\title{
Criteria for Floating I
}

\author{
Robert Finn
}

\begin{abstract}
Conditions are determined under which solid bodies will float on a liquid surface in stable equilibrium, under the influence of gravity and of surface tension. These include configurations in which the density of the body exceeds the density of the ambient liquid, so that for an infinitely deep liquid in a downward gravity field there is no absolute energy minimum. Of notable interest are the results (a) that if a smooth body is held rigidly and translated downward into an infinite fluid bath through a family of fluid equilibrium configurations in a downward gravity field, the transition is necessarily discontinuous, and (b) a formal proof that there can be a free-floating locally energy minimizing configuration that does not globally minimize, even if the density of the body exceeds that of the liquid. The present work is limited to the two dimensional case corresponding to a long cylinder that is floating horizontally. The more physical three-dimensional case can be studied in a similar way, although details of behavior can change significantly. That work will appear in an independent study written jointly with T. I. Vogel.
\end{abstract}

Mathematics Subject Classification (2000). 76B45, 49Q10, 53A10.

Keywords. Capillarity, surface tension, surface energy, floating bodies.

\section{Introduction}

There is a large and still developing literature on capillary surfaces in the presence of fixed rigid boundaries, based on concepts introduced during the early nineteenth century by Young, by Laplace and by Gauss. In contrast, the problem of determining the configuration of an isolated rigid body floating on a fluid surface, although discussed at some length by Laplace in his 1806 treatise [1] and clearly of great physical interest, has suffered nearly two centuries of neglect. I have been unable to locate any further literature on the topic prior to the past fifteen years, the earliest such work known to me being the elegant contribution [2] on a highly idealized case.

Floating bodies have not been entirely neglected; a number of papers of engineering character have appeared on the independent problem of describing interactions among distinct particles of ideally round shape and assumed to be floating, see, e.g. [3-6]. In the context of the evident interest in that material, the striking and clearly delineated mathematical results of [2] impressed on me the beauty of the problem and put into relief how little is known about the seemingly simplest situations. It motivated me to take up the question and to discuss it with others, leading to the contributions [7-11] relating to idiosyncratic behavior of the configurations that can appear. These are properties that have a clear physical interest but are expressed by geometrical rather than numerical description.

I know of no work in the classical literature, addressing the basic criteria for existence of physically meaningful configurations as solutions of underlying equations or physical principles, corresponding to prescribed conditions of body shape and weight, gravitational force, and interfacial energies (energy density associated with formation of surface interface). These problems are of special interest from the point of view of determining the suitability of the equations and methods as models of physical experience. A start has however been made in a recent paper by McCuan [12]. The present work offers an independent step to fill that gap, from another point of view. The results are in conceptual accord with what is expected, and also offer explicit estimates for critical parameters. The problem has a particular interest for the familiar case of a body whose density exceeds that of an infinitely deep liquid on which it is 


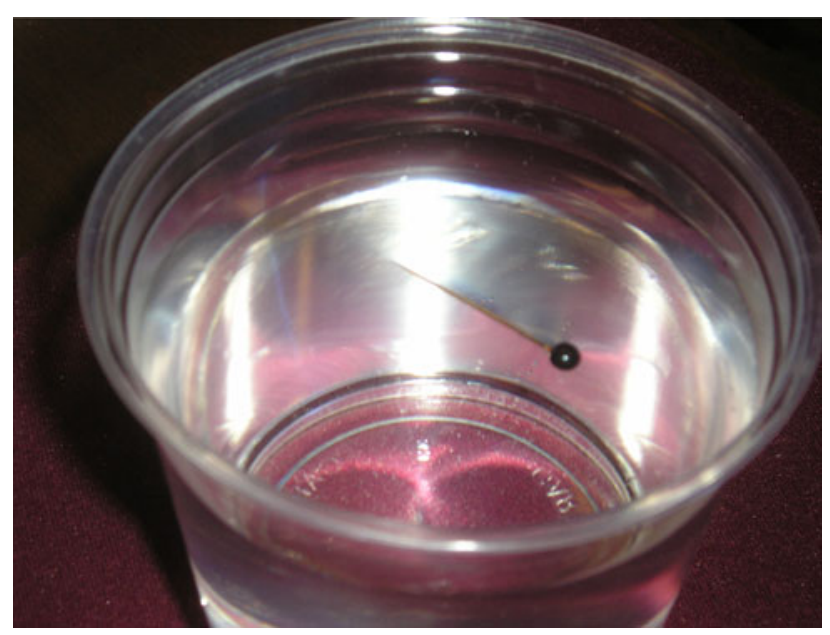

FIG. 1. Metal pin floating on water. The pin sinks if disturbed

floating, in a downward gravity field. That situation cannot provide a global minimum for the energy, as arbitrarily large negative energies are obtainable simply by submerging the body into the fluid. In the earth's gravity field the matter may not be of great importance for bodies of large size, such as a boat or even a duck, as then the surface tension effects will generally be small relative to those attributable to gravity. However the question acquires a new interest in the context of space exploration, in which surface tension forces generally prevail over gravitational effects, and also for microanalysis of small particle configurations on earth. The problems also take on special interest from the point of view of large numbers of (small) particles that may self-assemble themselves into organized patterns under appropriate conditions as has occasionally been observed, and also for the description of such phenomena as insects that "walk" on water. Also for larger objects, there can be physical interest in familiar circumstances, as indicated by the enclosed photograph (Fig. 1) of a metal pin that I was personally able (after several tries) to make float on a surface of tap water. Once achieved, that configuration turned out to be fairly stable, surviving moderate (albeit not severe) shaking.

A central feature of the results to follow is that the kinds of global behavior that can occur are severely limited by the constraints imposed on the shape of the ambient fluid surface, due to its surface tension in combination with the effects of bulk fluid weight. These properties of the interface separating the ambient media lead to behavior of partly immersed bodies that can differ qualitatively in striking ways from what is predicted when such effects are neglected. As a notable example, Archimedes' Law that a floating body displaces its weight in liquid loses in precision when surface tension is taken into account, and becomes expressed in terms of undefined quantities.

Corresponding to a supposed floating body configuration, we denote by $\mathrm{e}_{0}$ the surface energy distribution (or equivalently surface tension) in the interface $\mathcal{S}$ separating the ambient liquid from the outer medium (in general air or vacuo), by $\mathrm{e}_{1}$ the surface energy distribution of the body $\mathcal{B}$ with the outer medium, by $\mathrm{e}_{2}$ the corresponding distribution for $\mathcal{B}$ with the ambient liquid below $\mathcal{S}$. I assume in all cases that the fluid surface sufficiently far from the body is a graph $u(x, y)$ that tends to the level $u \equiv 0$ at infinity. For simplicity in this initial study, I will assume in most instances that $\mathcal{B}$ is strictly convex, in the sense indicated below. More general situations will be indicated by remarks in the text.

There can be great variety in behavior according to circumstance, and we must therefore distinguish individual cases. Our reasoning will be based on energy principles in conjunction with generally accepted physical laws. A formal difficulty appears owing to the infinite energy in the free surface of the ambient fluid. Since we are interested only in stationary points arising from local variations, we will restrict attention to equilibrium conditions arising from variations for which the energy changes can be clearly defined by limits of changes restricted to compact subsets. 


\section{Two Dimensions: $g=0$}

The simplest (although greatly idealized) case is that of a two-dimensional body $\mathcal{B}$ (section of an infinite horizontal cylinder) in zero gravity, $g=0$. We assume strict convexity in the sense that at each boundary point $P, \partial \mathcal{B}$ can be represented in local coordinates $(x, v(x))$, with $v(0)=v^{\prime}(0)=0, v^{\prime \prime}(0)>0$. We assume initially an everywhere horizontal fluid surface $\mathcal{S}: u(x) \equiv 0$ in mechanical equilibrium in the absence of disturbing forces, and we seek criteria under which $\mathcal{B}$ can be partly submerged in a configuration of locally minimizing mechanical energy. We begin by asking what happens when $\mathcal{B}$ is held rigidly in a fixed orientation and gradually lowered from positive infinity toward $\mathcal{S}$, as in Fig. 2. Denoting $|\partial \mathcal{B}|$ by s $\mathcal{B}$ we examine the energy $\mathcal{E}$ as function of height $h$ and obtain:

Lemma 1.1. $\mathcal{S}$ remains unchanged prior to contact with $\mathcal{B}$. In the range $h_{2}<h<h_{1}$ of heights for which $\mathcal{B}$ contacts $\mathcal{S}, \mathcal{S}$ remains at the same horizontal level, but with the segment of intersection with $\mathcal{B}$ deleted (Fig. 3). If $\left|\frac{\mathrm{e}_{2}-\mathrm{e}_{1}}{\mathrm{e}_{0}}\right|>1$ then $\mathcal{E}^{\prime}(h) \neq 0$ throughout $\left(h_{2}, h_{1}\right)$. If $\left|\frac{\mathrm{e}_{2}-\mathrm{e}_{1}}{\mathrm{e}_{0}}\right|<1$ then there is a unique height at which $\mathcal{E}^{\prime}(h)=0$, and at this height the configurational energy achieves an absolute minimum over the range of vertical positions, for the given orientation.

Proof. By Pascal's Law the pressures above and below $\mathcal{S}$ are each constant throughout those portions of the fluids, hence the pressure change $\delta p$ across $\mathcal{S}$ is the same at all points of $\mathcal{S}$. By a discovery of Young [13], $\delta p=\mathrm{ke}_{0}$, where $\mathrm{k}$ is the curvature of $\mathcal{S}$. Thus $\mathcal{S}$ has constant curvature and must be a circular arc. Since at plus and at minus infinity $\mathcal{S}$ has the constant height $u=0$, the only possibility is that $\mathcal{S}$ is the horizontal line (degenerate arc) $u \equiv 0$. This result is independent of the height of $\mathcal{B}$ above $\mathcal{S}$, that is, regardless of the attractions of $\mathcal{B}$ (or of other eventual rigidly held objects) for the fluid particles, and in that sense could be viewed as physically unrealistic.

When $\mathcal{B}$ is partially immersed in the liquid, the energy $\mathcal{E}$ can be written up to a constant in the form:

$$
\mathcal{E}=\mathrm{e}_{1} \mathrm{~s}_{1}+\mathrm{e}_{2} \mathrm{~s}_{2}-\mathrm{e}_{0} \mathrm{~s}_{0}
$$

with $\mathrm{s}_{1}$ and $\mathrm{s}_{2}$ the relative portions of $\mathrm{s}_{\mathcal{B}}$ in the upper and lower fluids, so that $\mathrm{s}_{1}+\mathrm{s}_{2}=\mathrm{s}_{\mathcal{B}}$. Writing $\mathrm{s}_{j}=\lambda_{j} \mathrm{~s}_{\mathcal{B}}$ for $j=0,1,2$, and noting first that $\lambda_{1}+\lambda_{2}=1$ and then that $\lambda_{0}$ is a function of $\lambda_{2}, \lambda_{0}=f\left(\lambda_{2}\right)$, we can write

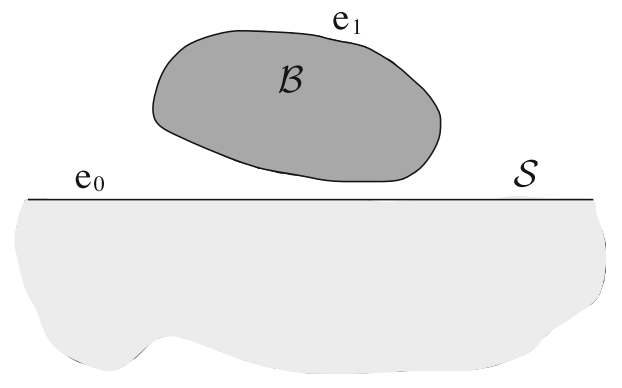

FIG. 2. Configuration prior to contact with $\mathcal{S}$.

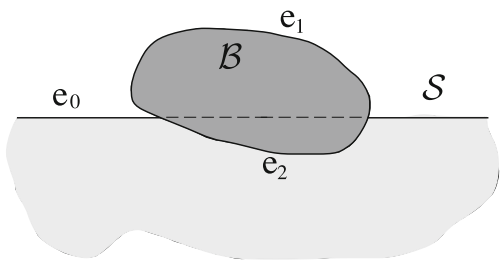

FIG. 3. Configuration after initial contact with $\mathcal{S}$. 


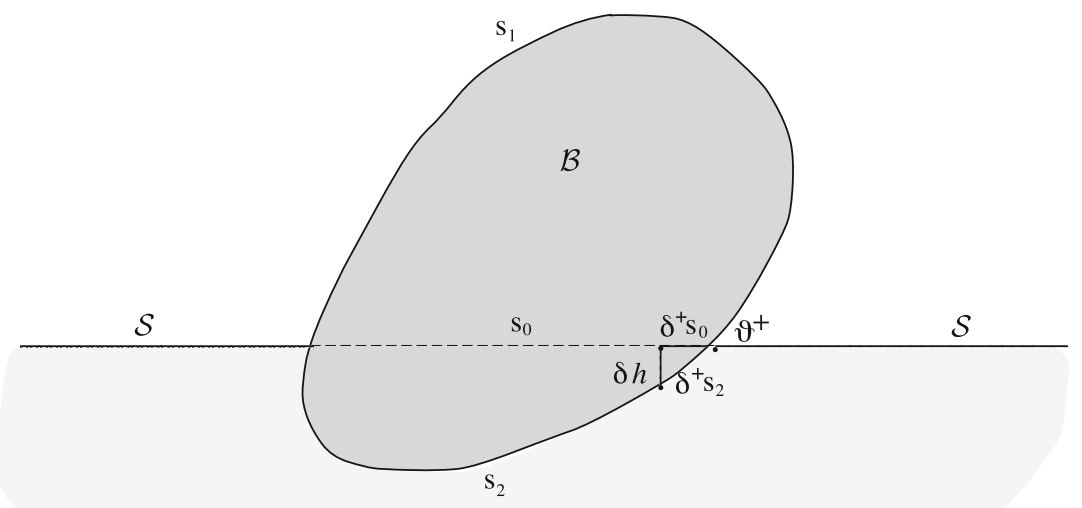

FIG. 4. Dipping relations.

$$
\mathcal{E}_{\mathcal{B}} \equiv \frac{1}{\mathrm{~s}_{\mathcal{B}}} \mathcal{E}=\left(1-\lambda_{2}\right) \mathrm{e}_{1}+\lambda_{2} \mathrm{e}_{2}-f\left(\lambda_{2}\right) \mathrm{e}_{0},
$$

defining $\mathcal{E}$ as a function of $\lambda_{2}$, for which equilibrium points are obtained from

$$
f^{\prime}\left(\lambda_{2}\right)=\frac{\mathrm{e}_{2}-\mathrm{e}_{1}}{\mathrm{e}_{0}}
$$

In view of the convexity of $\mathcal{B}$, we find that $f^{\prime}\left(\lambda_{2}\right)$ is continuous in the range $0<\lambda_{2}<1$, and $-1<$ $f^{\prime}\left(\lambda_{2}\right)<1$ in that range, with $f^{\prime}(0)=1, f^{\prime}(1)=-1$, see Fig. 3. Thus if $\left|\frac{\mathrm{e}_{2}-\mathrm{e}_{1}}{\mathrm{e}_{0}}\right|>1$ then there is no equilibrium configuration for which points of (the extended) $\mathcal{S}$ strictly enter $\mathcal{B}$, and $\mathcal{E}$ changes monotonically from $\mathrm{e}_{1} \mathrm{~s}_{\mathcal{B}}$ to $\mathrm{e}_{2} \mathrm{~s}_{\mathcal{B}}$. However, if $\left|\frac{\mathrm{e}_{2}-\mathrm{e}_{1}}{\mathrm{e}_{0}}\right|<1$ there is at least one value of $\lambda_{2}$ in the range, for which (1.3) holds and provides equilibrium.

We can say more. Denoting by $\vartheta^{+}$and $\vartheta^{-}$the incidence angles of $\partial \mathcal{B}$ with $S_{0}$ (see Fig. 4 ), we find

$$
\begin{aligned}
& \delta \mathrm{s}_{2}=\left(\csc \vartheta^{-}+\csc \vartheta^{+}\right) \delta h \\
& \delta \mathrm{s}_{0}=\left(\cot \vartheta^{-}+\cot \vartheta^{+}\right) \delta h
\end{aligned}
$$

so that, denoting differentiation in $\lambda_{2}$ by $(\cdot)$,

$$
f^{\prime}\left(\lambda_{2}\right) \equiv \dot{\lambda}_{0}=\frac{\sin \left(\vartheta^{-}+\vartheta^{+}\right)}{\sin \vartheta^{-}+\sin \vartheta^{+}} .
$$

From (1.6) we compute

$$
\ddot{\lambda}_{0}=-\frac{\left(\dot{\vartheta}^{-} \sin \vartheta^{+}+\dot{\vartheta}^{+} \sin \vartheta^{-}\right)}{\left(\sin \vartheta^{-}+\sin \vartheta^{+}\right)^{2}}\left[1-\cos \left(\vartheta^{-}+\vartheta^{+}\right)\right] .
$$

From (1.2) we have

$$
\ddot{\mathcal{E}}_{\mathcal{B}}=-\mathrm{e}_{0} \ddot{\lambda}_{0}
$$

Since both numerator terms in (1.7) are positive by the convexity of $\mathcal{B}$, we conclude that $f^{\prime \prime}\left(\lambda_{2}\right)<0$; thus the critical value is unique and provides a global minimum for the energy. By (1.4), $h$ is strictly monotone in $\mathrm{s}_{2}$; thus, there is a unique value of $h$ at which the minimum is achieved, as asserted. 
We have incidentally proved the general geometrical statement:

Corollary. Let $\mathcal{B}$ be a strictly convex plane domain, and let $\mathcal{L}$ be a support line of $\mathcal{B}$. The length of the chords intercepting $\mathcal{B}$ and parallel to $\mathcal{L}$ is a strictly concave function of the boundary lengths cut off by the chords, and thus there is a unique such chord that achieves a maximum length.

The relative minimum obtained in the above procedure cannot be expected to have a physical significance relative to freely floating bodies; for example, there is no contact angle condition. Even were the physical contact angle to be attained on one side of $\mathcal{B}$, in general it will not be attained on the other side, and we are not free to adjust the liquid surface to achieve a desired angle of incidence. We are however free to change the orientation $\omega_{B}$ of $\mathcal{B}$, which we have taken till now as prescribed. In this respect we note that the criterion (1.3) depends only on the choice of materials and is independent of orientation. We thus obtain:

Lemma 1.2. The determination as to whether a minimum for the energy is attained during contact of $\mathcal{B}$ with $\mathcal{S}$ is independent of the orientation of $\mathcal{B}$.

The orientations available are restricted to the compact set $0 \leq \omega_{B} \leq 2 \pi$, and the associated minimizing energies are a continuous function on that set. Thus there exists at least one choice $\omega_{\mathcal{B}}$ leading to a least possible minimal energy. If we denote by $h_{c}$ the height of the centroid $c$ of $\mathcal{B}$ over the reference level $z=0$, we find:

Theorem 1.1. If $\left|\frac{\mathrm{e}_{2}-\mathrm{e}_{1}}{\mathrm{e}_{0}}\right|<1$, there exists a height $h_{c}$ and corresponding orientation $\omega_{\mathcal{B}}$, for which an absolute minimum $\mathcal{E}_{m}$ of $\mathcal{E}$ is achieved, over all heights and orientations. For any such configuration, $\mathcal{S}$ abuts on $\mathcal{B}$.

Corollary. For the height and orientation indicated in Theorem 1.1, the "unseen hand" that holds $\mathcal{B}$ rigidly can be removed, and $\mathcal{B}$ will be in a "free-floating" equilibrium position of minimizing energy.

For a configuration achieved in this way, we find:

Theorem 1.2. (Gauss criterion). In a minimizing configuration as described in Theorem $1.1, \mathcal{B}$ is positioned so that its boundary meets $\mathcal{S}$ at both intersection points in the angle $\gamma$ (measured within the lower medium) for which

$$
\cos \gamma=\frac{\mathrm{e}_{2}-\mathrm{e}_{1}}{\mathrm{e}_{0}}
$$

Proof. Referring to the configuration as in Fig. 3, we make a variation by rotating $\mathcal{B}$ about one of the points $P$ of intersection with $\mathcal{S}$ (Fig. 5).

For the energy change, we obtain

$$
\delta \mathcal{E} \cong\left(\mathrm{e}_{2}-\mathrm{e}_{1}\right) \delta \mathrm{s}-\mathrm{e}_{0} \delta \mathrm{s}_{0}=\left[\left(\mathrm{e}_{2}-\mathrm{e}_{1}\right)-\mathrm{e}_{0} \cos \vartheta\right] \delta \mathrm{s}
$$

and thus equilibrium imposes (1.9), since $\vartheta=\pi-\gamma$.

Note that the proof involved a rotation of $\mathcal{B}$, cf. the remarks just below.

Minimizing configurations are in general not unique. For example, it is shown in [2] that for convex $\mathcal{B}$ at least four distinct equilibrium positions exist; if $\mathcal{B}$ is elliptical but not a circle, then there are exactly four, of which two are local minima for the energy (it should be noted that relative to the vertical motions introduced above, all provide minima; thus, an ellipse placed in an unstable equilibrium position and released will rotate rather than translate). If $\mathcal{B}$ is a circular disk, then all orientations minimize at the same height. In [10] non-circular sections $\mathcal{B}$ are characterized that admit continua of angular width $\pi$, of minimizing orientations.

Physically, the case $\left|e_{2}-e_{1}\right|>e_{0}$ corresponds either to the situation in which fluid covers the entire ball, or else to the case in which fluid separates from the ball completely, according as $\mathrm{e}_{2}-\mathrm{e}_{1}$ is negative or positive. These configurations are not addressed in the present work.

As already remarked, the hypotheses introduced above are in some ways physically unrealistic. We proceed step by step to relax them. 


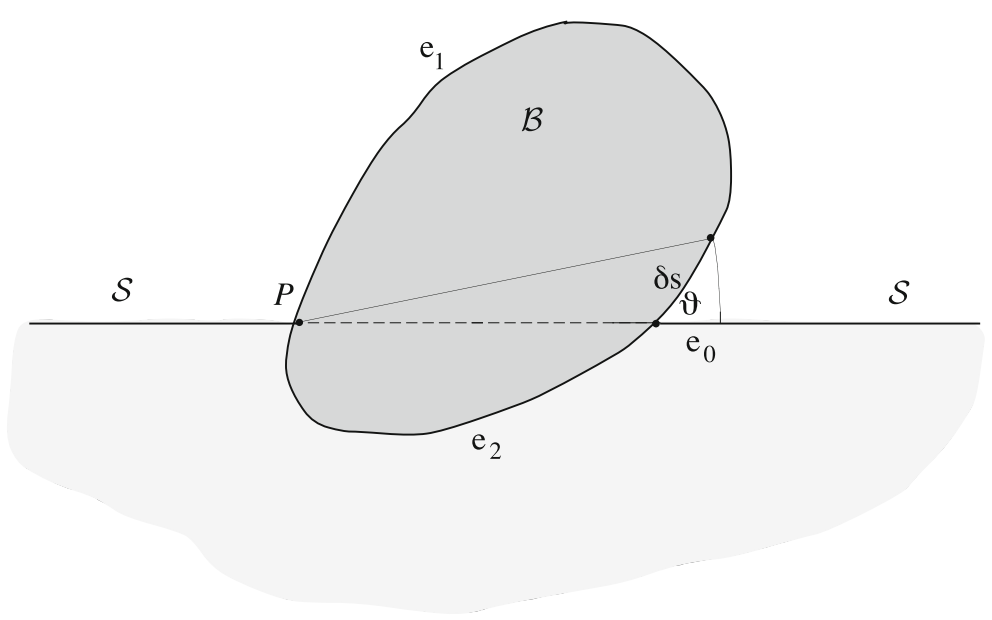

FIG. 5. Energy variation by rotation.

\section{Two Dimensions: $g>0$}

By $g>0$ is meant a gravity field of strength $g$ per unit mass directed downward across the interface. The surface $\mathcal{S}$ is no longer constrained to be flat, however its surface tension in conjunction with the gravity field severely constrains its behavior. From the basic equation for $\mathcal{S}$ in terms of its inclination $\psi$, which goes back to Young and to Laplace

$$
(\sin \psi)_{x}=\kappa u
$$

where $\kappa=\rho g / \sigma$ with $\rho=$ density change across $\mathcal{S}$ and $\sigma=$ surface tension of $\mathcal{S}$, we can write

$$
(\sin \psi)_{u} \tan \psi=\psi_{u} \sin \psi=\kappa u
$$

which leads to the parametric representation

$$
\begin{aligned}
& x=x_{0}+\frac{1}{\sqrt{2 \kappa}} \int_{0}^{\varphi} \frac{\sin \varphi}{\sqrt{1-\sin \varphi}} d \varphi \\
& u=\sqrt{\frac{2}{\kappa}(1-\sin \varphi)}
\end{aligned}
$$

of the solution curve in terms of $\varphi=(\pi / 2)-\psi$. In the present instance we impose the condition $u=0(\varphi=\pi / 2)$ at $x=\infty$. The point $x=x_{0}, u=\sqrt{2 / \kappa}$ is vertical on the curve, but the curve can be continued beyond that point till a maximum height $u_{M}=2 / \sqrt{\kappa}$ at $\varphi=-\pi / 2$, doubly covering an interval on the $x$-axis. We see that $\mathcal{S}$ is completely determined, up to translation along the $x$-axis and reflection in the $x$ or $y$ axes. Continuation beyond the maximum point produces no significantly new trajectory points, but instead only the identical curve reflected in that value of $x$. See Fig. 6, which is taken from the treatise [14] of Bakker. These limitations on the behavior of $\mathcal{S}$ are essential in the estimates to follow.

We have immediately:

Theorem 2.1. If the density of $\mathcal{B}$ differs from that of the upper ambient medium, then no equilibrium can occur for which $\mathcal{B}$ lies entirely above the level $2 / \sqrt{ } \kappa$. If the density of $\mathcal{B}$ differs from that of the lower ambient medium, then no equilibrium can occur for which $\mathcal{B}$ lies entirely below the level $-2 / \sqrt{ } \kappa$.

Proof. The universal bound $|u|<2 / \sqrt{ } \kappa$ for $\mathcal{S}$ precludes contact of $\mathcal{S}$ with $\mathcal{B}$ in the envisaged configurations, and thus if $\mathcal{B}$ lies as indicated then $\mathcal{S}$ must be the undisturbed line $u \equiv 0$, which is the only global solution of (2.1) that vanishes at $x= \pm \infty$. For $\mathcal{B}$ either above or below that line, the surface energy 


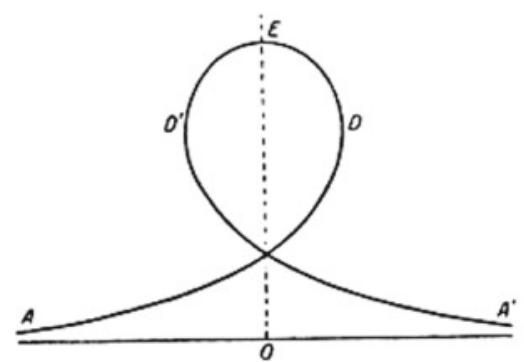

FIG. 6. The surface $\mathcal{S}$, extending from $-\infty(A)$ to the vertical $D$ at height $\sqrt{2 / \kappa}$, then turning back and covering doubly to a horizontal $\mathcal{E}$ at height $2 / \sqrt{\kappa}$. This curve is then reflected in $x=0$ to obtain the continuation to the vertical $D^{\prime}$ and then to $+\infty\left(A^{\prime}\right)$.

remains constant independent of position. For the gravitational energy we obtain, for $\mathcal{B}$ of density $\rho$ and situated in the upper medium of density $\rho_{1}$ :

$$
\begin{aligned}
\mathcal{E}_{g} & =g \mathrm{~s}_{\mathcal{B}}\left(\rho-\rho_{1}\right) \int_{h^{-}}^{h^{+}} y \lambda_{0}(y) d y \\
& =g \operatorname{s}_{\mathcal{B}}\left(\rho-\rho_{1}\right)\left\{\int_{h^{-}}^{h^{+}}\left(y-h^{-}\right) \lambda_{0}(y) d y+h^{-} \int_{h^{-}}^{h^{+}} \lambda_{0}(y) d y\right\} \\
& =g \mathrm{~S}_{\mathcal{B}}\left(\rho-\rho_{1}\right)\left(C+C_{1} h^{-}\right)
\end{aligned}
$$

where $C, C_{1}>0$ are constants depending only on the shape of $\mathcal{B}$, and where $h^{-}$and $h^{+}$are the lower and upper heights on $\mathcal{B}$. If $\mathcal{B}$ is in the lower medium of density $\rho_{2}$, then

$$
\mathcal{E}_{g}=g_{\mathrm{s}_{\mathcal{B}}}\left(\rho-\rho_{2}\right)\left(C+C_{1} h^{-}\right)
$$

Under the hypotheses, in both cases, the energy would then be proportional to height of $\mathcal{B}$, precluding equilibrium.

Note that the values $h^{-}$in (2.3) and in (2.4) differ; the former is positive, the latter negative.

Theorem 2.2. Let a homogeneous body $\mathcal{B}$ with smooth boundary be moved rigidly in any continuous way from a position in which $\mathcal{B}$ lies above the level $2 / \sqrt{ } \kappa$ to one in which $\mathcal{B}$ lies below the level $-2 / \sqrt{ } \kappa$ Then if $g>0$, there must be a discontinuous change in the configuration of $\mathcal{S}$ in the course of the traverse.

Proof. As $\mathcal{B}$ moves downward and into the strip between the two levels but prior to crossing the level $u=0$, a configuration conceivably appears in which $\mathcal{S}$ rises to contact $\mathcal{B}$ with locally stationary energy (cf. Fig. 8). Such a change would have to occur discontinuously, as the only $\mathcal{S}$ satisfying the conditions at infinity and not meeting $\mathcal{B}$ is the line $u \equiv 0$. Alternatively, $\mathcal{S}$ could remain undisturbed until a point $P \in \mathcal{B} \cap \mathcal{S}$ first appears, as in the left side of Fig. 7. At such a point, $\mathcal{S}$ meets $\mathcal{B}$ with contact angle $\gamma=\pi$, see Fig. 7. We make a variation, replacing the segment $\delta x$ by $\delta y$, and allowing the fluid below $\mathcal{S}$ to rise up to $\mathcal{B}$ below $\delta$ s. We find for the change of surface energy

$$
\delta \mathcal{E}_{s} \simeq\left(\mathrm{e}_{2}-\mathrm{e}_{1}\right) \delta s-\mathrm{e}_{0}(\delta x-\delta y)=\left(\mathrm{e}_{2}-\mathrm{e}_{1}-\mathrm{e}_{0}\right) \delta s+o(\delta s)
$$

in view of the cusp at $P$, while the gravitational energy change $\delta \mathcal{E}_{g}=o(\delta \mathrm{s})$, since due to the cusp at $P$ the change in fluid area is $o(\delta s)$. Thus, the configuration for initial contact is not stationary for the energy unless

$$
\mathrm{e}_{2}-\mathrm{e}_{1}=\mathrm{e}_{0}
$$

in agreement with (1.9) for $\gamma=\pi$. 


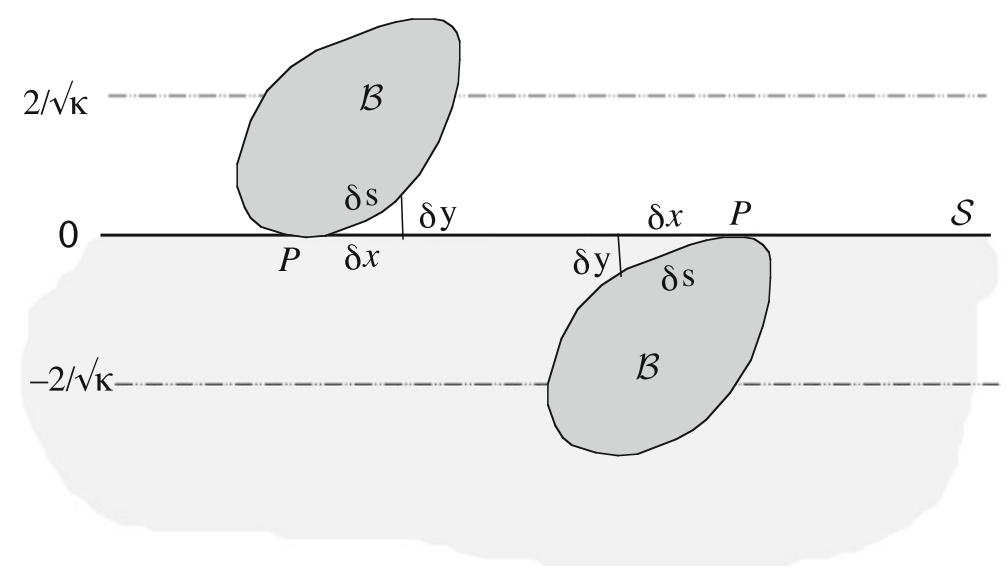

FIG. 7. Configurations of initial and of final contact with the horizontal $\mathcal{S}$.

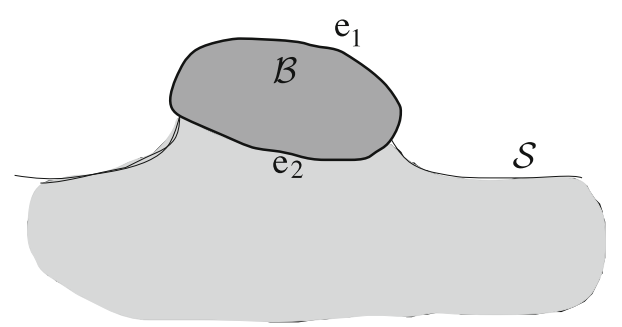

FIG. 8. To achieve minimum energy when $\mathrm{e}_{2}-\mathrm{e}_{1} \neq \mathrm{e}_{0}$, fluid must rise discontinuously to meet $\mathcal{B}$.

If (2.6) fails then the configuration must jump discontinuously to a distinct equilibrium state. If (2.6) holds, the given configuration will be in equilibrium and may change continuously as $\mathcal{B}$ is lowered further, initially intercepting $\mathcal{S}$. If the change remains continuous, there will be a minimum height at which contact still occurs, as indicated on the right side of Fig. 7. At this height one has $\gamma=0$, and reasoning analogous to that leading to (2.6) now yields

$$
\mathrm{e}_{1}-\mathrm{e}_{2}=\mathrm{e}_{0}
$$

This is in conflict with (2.6); we conclude that such a configuration cannot appear, and thus at some intermediate position a discontinuous jump in the fluid surface heights must have occurred.

Remark. If (2.6) fails, then as $\mathcal{B}$ is lowered from above and becomes sufficiently close to-although still above - the horizontal $\mathcal{S}$, a configuration with $\mathcal{S}$ abutting on $\mathcal{B}$ must appear that has lower energy than the one with horizontal $\mathcal{S}$. That is because the energy prior to contact approaches the energy with initial contact, whereas energy decreases discontinuously when initial contact occurs, see Fig. 8. Nevertheless, so long as $\mathcal{B}$ remains strictly above $\mathcal{S}$, the configuration with $\mathcal{S}$ horizontal and disjoint from $\mathcal{B}$ is locally stable.

\section{An Illustration}

The following material is somewhat peripheral to the essential substance of this paper, and is included to illustrate the theorems directly above in the explicit case for which $\mathcal{B}$ is a rectangle, and thus a limiting case of the strictly convex shapes considered till now. We assume again $g>0$ and position $\mathcal{B}$ with its sides 


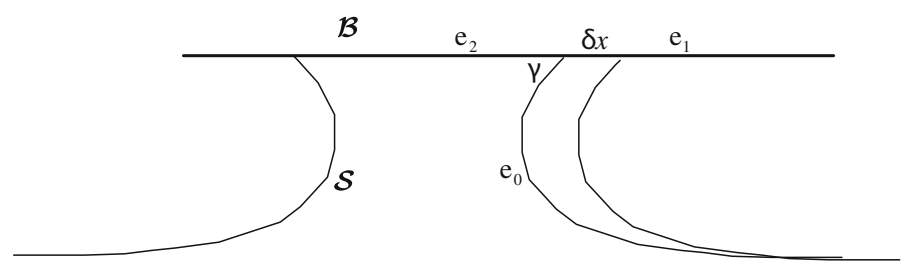

FIG. 9. Fluid cannot rise to the interior of a horizontal bottom.

parallel and perpendicular to the horizontal. Our initial observation can already be found in Theorem 1.1 of Vogel [15], in another connection.

Assertion 3.1. Any equilibrium configuration in which fluid rises to a part of $\partial \mathcal{B}$ that is horizontal as in Fig. 9 is unstable, in the sense that energy can be decreased by a smooth configuration path over which energy is non-increasing.

Proof. The fluid boundary $\mathcal{S}$ rising to $\mathcal{B}$ is contained in the family (2.2). The Gauss criterion (1.9) is easily shown to hold at the contact point, and thus for the respective energy densities

$$
\mathrm{e}_{1}=\mathrm{e}_{2}+\mathrm{e}_{0} \cos \gamma
$$

The surface $\mathcal{S}$ continues to be described by (2.2) if it is shifted rigidly to the right a horizontal distance $\delta x$, as indicated in Fig. 9. The change in surface energy encountered in this shift is

$$
\delta \mathcal{E}_{S}=\left(\mathrm{e}_{2}-\mathrm{e}_{1}-\mathrm{e}_{0}\right) \delta x,
$$

the term involving $\mathrm{e}_{0}$ appearing since the energy change occasioned by shifting $\mathcal{S}$ is $\mathrm{e}_{0}$ times the change in length of $\mathcal{S}$, which can be evaluated at infinity where $\mathcal{S}$ is horizontal. To estimate the change in gravitational energy we observe that the region between $\mathcal{S}$ and its shifted image is generated by horizontal segments of width $\delta x$. The work done in raising a vertical interval of liquid in that region from height zero to height $h$ is $\rho g h \delta h \delta x$, and thus the gravitational energy within the region is

$$
\delta \mathcal{E}_{g}=\frac{1}{2} \rho g u^{2} \delta x=\frac{1}{2} \rho g\left(\frac{2 \sigma}{\rho g}(1-\sin \varphi)\right) \delta x=\mathrm{e}_{0}(1+\cos \gamma) \delta x
$$

by (2.2), since on a fluid interface, surface tension is equivalent to energy density (see, e.g. [16]). Thus, the net energy change is

$$
\delta \mathcal{E}=\delta \mathcal{E}_{S}+\delta \mathcal{E}_{g}=\left(\mathrm{e}_{2}-\mathrm{e}_{1}+\mathrm{e}_{0} \cos \gamma\right) \delta x=0
$$

by (3.1). It follows that without changing the energy, we can move the two fluid boundaries together until they touch, as indicated in Fig. 9. Having done that, removing the fluid above the horizontal surface and bounded between the two parts of $\mathcal{S}$ that extend to infinity will lower the energy. We are done.

Assertion 3.2. There exist configurations in which $\mathcal{B}$ lies strictly above the ambient level, but in which the energy is less than that occurring for undisturbed fluid.

Proof. We fix $\kappa>0$ and contact angle $\gamma, 0<\gamma<\pi / 2$ We immerse $\mathcal{B}$ vertically, as in Fig. 8 , and observe that if its height exceeds $2 / \sqrt{ } \kappa$ it can be positioned so that its top and bottom are at heights above and below the heights reached by $\mathcal{S}$ at its vertical points. Since the slope of $\mathcal{S}$ is monotone in $x$, it follows that among the solutions (2.2) there will then be a unique (symmetric) pair that meet $\mathcal{B}$ in angle $\gamma$ on its vertical sides, as in Fig. 9. Any other pair that abut in $\mathcal{B}$ (without regard to angle) is obtained from the given one by rigid horizontal shifting of $\mathcal{S}$ (Figs. 10,11).

Within this family of solutions, a variational reasoning shows that the unique pair abutting in angle $\gamma$ provides a strict absolute minimum for the energy, corresponding to a fixed position for $\mathcal{B}$. 


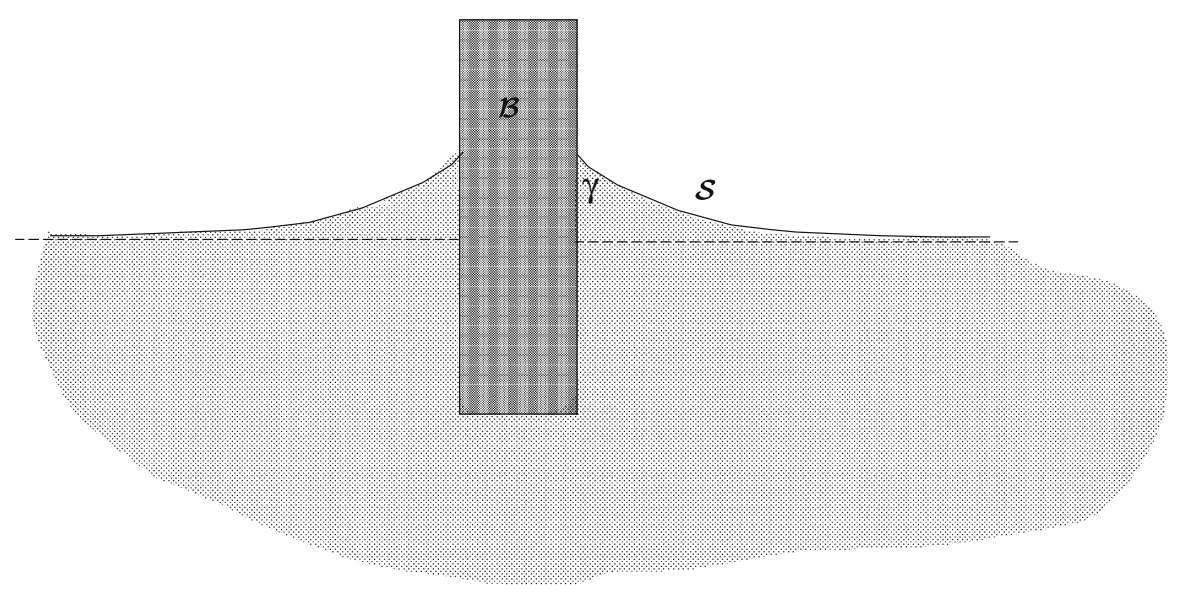

FIG. 10. Theorem 2.3: initial steps.

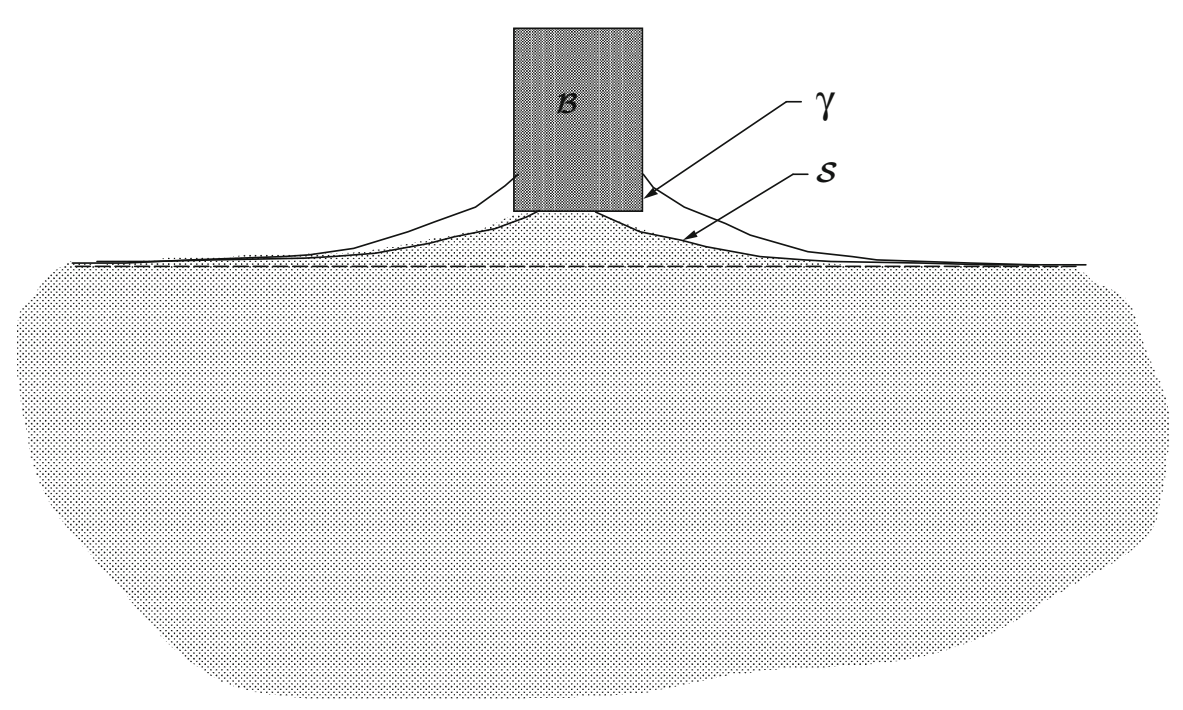

FIG. 11. Theorem 2.3: final steps.

Now shorten $\mathcal{B}$ by drawing a horizontal segment $L$ above its base but not above the ambient level, and then removing the portion of $\mathcal{B}$ below $L$. That changes the total energy of the system, but does not affect the minimizing property of the $\mathcal{S}$ we have chosen, as no new surfaces can enter into competition.

On raising $\mathcal{B}$ so that $L$ is at the ambient position, we thus find that the energy $\mathcal{E}$ is strictly less (in the appropriate comparison sense) than that arising from the horizontal surface $\mathcal{S}_{0}$ exterior to $\mathcal{B}$, plus that of $L$. But the change in energy going from this latter configuration to that of the completed planar fluid surface and also an $L$ slightly above that surface, is $\left(\mathrm{e}_{1}-\mathrm{e}_{2}\right) L+\mathrm{e}_{0} L$ which is positive in view of (3.1), since $\cos \gamma>0$. So the configuration we have found remains minimizing for curves meeting the sides of $\mathcal{B}$, even after $\mathcal{B}$ is lifted slightly out of the fluid.

We are not quite done, as we must still consider eventual fluid surfaces that abut on $L$ after it is lifted from the fluid. It is conceptually convenient here to round the lower corners of $\mathcal{B}$, with circular arcs of radius small enough not to affect the energy inequality just established. We may clearly restrict the competing surfaces to members of the family (2.2), and we note that if $L$ is close to the ambient level 
then these curves will be restricted to have arbitrarily small slopes, and thus no new equilibrium configurations corresponding to the given $\gamma$ will appear. The single danger point occurs when the solutions from opposite sides meet at a common point of $L$. But the energy of such a configuration exceeds that in which the fluid surfaces are replaced by the ambient level, and we have just shown that energy exceeds that of the given extremal configuration. The proof is complete.

We note that if $\mathcal{B}$ is moved down slowly from a high level toward the lower fluid, the situation of Assertion 3.2 can only occur via a discontinuous jump passing through surfaces in which energy initially rises. That is so since a smooth change would have to be established via intermediate surfaces that lie entirely below $\mathcal{B}$, and these all provide energies exceeding those of both the starting configuration with horizontal $\mathcal{S}$ disjoint from $\mathcal{B}$, and of the final configuration with $\mathcal{B}$ globally minimizing.

\section{Floating Criteria: $g>0$}

We now return to our original hypothesis of general convex $\mathcal{B}$, of prescribed density and in a prescribed gravity field. We hold its centroid rigidly as we move it downward with height $h_{c}$ from infinity, allowing orientation to adjust at each height to a minimizing position. We seek configurations, in which a stable equilibrium will be found, that is, we seek configurations that will be mechanically stable when the unseen hand holding the centroid is removed.

We postpone the case in which $\mathcal{B}$ has smaller density than the upper medium, and proceed to the next case, in which $\rho_{2}<\rho<\rho_{2}$. Since surface energy is constant for all positions for which $\mathcal{B}$ lies entirely within one of the fluids, we see from (2.3) and (2.4) that the energy functional becomes positive infinite, both as $\mathcal{B}$ rises to $+\infty$ and as $\mathcal{B}$ descends to $-\infty$. Assuming continuity of that functional with position, it is thus clear that at least one minimizing configuration will exist. For convex $\mathcal{B}$, continuity can always be achieved by keeping the shape of $\mathcal{S}$ fixed as $\mathcal{B}$ is moved through it; the actual minimum cannot exceed the one achieved by such a motion. We can assert further that for any minimizing configuration, $\mathcal{B}$ contacts the free surface $\mathcal{S}$, as otherwise moving $\mathcal{B}$ upwards (or downwards) would (by (2.3) and (2.4)) decrease energy until contact occurs. More specifically we assert:

Theorem 4.1. If $\rho_{1}<\rho<\rho_{2}$ then there is at least one position of minimizing energy. If $\left|\mathrm{e}_{2}-\mathrm{e}_{1}\right| / \mathrm{e}_{0}<1$ then for every such position the angle $\gamma$ at the two contact points in which $\mathcal{B}$ meets $\mathcal{S}$ is determined by (3.1).

We have shown everything up to the angle condition. That assertion follows by a procedure analogous to the proof of Theorem 1.2.

We proceed now to the case of special interest, for which $\rho_{1}<\rho_{2}<\rho$. Then for an infinitely deep reservoir, the energy can be made arbitrarily large negative by moving $\mathcal{B}$ downwards, and thus it is clear that no absolute minimum for energy can be found. Nevertheless, it can happen that surface energies conspire to hold the body in floating configuration, at which a locally stable equilibrium appears for the energy.

Theorem 4.2. Let $\mathcal{B}$ be a prescribed convex body of density $\rho>\rho_{2}>\rho_{1}$ in a given downward gravity field $g$, and let $\mathrm{e}_{0}$ be the fluid/fluid interfacial tension. Then depending on $\mathcal{B}$ and on the indicated parameters, there are choices of $\mathrm{e}_{0}, \mathrm{e}_{1}$ and $\mathrm{e}_{2}$ so that an energy minimum will appear that is not global, and in which $\mathcal{B}$ meets $\mathcal{S}$.

Proof. Denote by $h_{c}^{+}$a centroid height such that for all possible orientations of $\mathcal{B}$ such that $h_{c}=h_{c}^{+}, \mathcal{S}$ will lie in its rest position $u \equiv 0$ with $\mathcal{B}$ strictly above it; by Theorem 2.1 it suffices to choose $h_{c}^{+}=$ $2 \sqrt{\mathrm{e}_{0} /\left(\rho_{2}-\rho_{1}\right) g}+d$, where $d$ is the diameter of $\mathcal{B}$. Similarly we may introduce $h_{c}^{-}=2 \sqrt{\mathrm{e}_{0} /\left(\rho_{2}-\rho_{1}\right) g}-d$, so that $\mathcal{S}$ will be in rest position with $\mathcal{B}$ strictly below it. Let $M$ be the maximum of gravitational energies among all orientations of $\mathcal{B}$, such that $h_{c}=h_{c}^{+}$, and let $m$ be the minimum of gravitational energies of $\mathcal{B}$, 
such that $h_{c}=h_{c}^{-}$. Using (2.3) and (2.4) we find that $M<A_{1}+B_{1} \sqrt{ } \mathrm{e}_{0}$, while $m>A_{2}-B_{2} \sqrt{ } \mathrm{e}_{0}$, where the $A$ and $B$ terms depend only on gravitational quantities, and not on surface energies.

Starting with $h_{c}=h_{c}^{+}$, we insert an unseen hand to gradually lower $h_{c}$, at each height adjusting the orientation so as to achieve a minimum $\mathcal{E}\left(h_{c}\right)$ for the total energy. A minimum always exists, as the entire configuration is controlled by a finite number of parameters, lying in a compact set. As noted above (Theorem 2.3) we must expect that at particular heights $h_{c}$ the energy change will occur discontinuously, in the sense of discontinuous changes in orientation of $\mathcal{B}$ or the configuration of $\mathcal{S}$. However, given a sequence of heights $h_{c}^{j} \longrightarrow h_{c}^{0}$ and orientations $\omega_{c}^{j} \longrightarrow \omega_{c}^{0}$, and letting $\mathcal{E}_{0}=g l b \mathcal{E}$ as those values are approached, we conclude from the compactness of the parameter domain that there exists a fluid configuration corresponding to $\left(h_{c}^{0} ; \omega_{0}^{c}\right)$ for which $\mathcal{E}_{0}$ is achieved. That configuration need not be unique.

We choose $\mathrm{e}_{0}$ sufficiently large that $(M-m) / \mathrm{e}_{0} \mathrm{~s}_{\mathcal{B}}<1$. We now note that the change in surface energy from the $h_{c}^{+}$position to the $h_{c}^{-}$position is exactly $\left(\mathrm{e}_{2}-\mathrm{e}_{1}\right) \mathrm{s}_{\mathcal{B}}$; thus if we choose $\mathrm{e}_{1}$ and $\mathrm{e}_{2}$ in any way such that $\left(\mathrm{e}_{2}-\mathrm{e}_{1}\right) \mathrm{s}_{\mathcal{B}}>M-m$ then the total energy of the configuration will increase from the upper to the lower position. Having made such a choice, we then scale $\mathrm{e}_{1}$ and $\mathrm{e}_{2}$ by a common factor to achieve

$$
1>\frac{\mathrm{e}_{2}-\mathrm{e}_{1}}{\mathrm{e}_{0}}>\frac{M-m}{\mathrm{~s}_{B} \mathrm{e}_{0}}
$$

In the initial configuration with $\mathcal{B}$ above $\mathcal{S}$, the energy initially decreases as $h_{c}$ is lowered, and it will continue to decrease until a height is reached at which the total energy with $\mathcal{S}$ flat equals the energy of a configuration in which $\mathcal{S}$ has jumped up discontinuously and abuts on $\mathcal{B}$, as in Fig. 8 . This is a configuration of non-uniqueness, as indicated above.

Further decrease of $h_{c}$ may lead to still smaller energies, and ultimately results in the configuration with $h_{c}=h_{c}^{-}$and $\mathcal{B}$ below $\mathcal{S}$, as constructed above, with total energy exceeding the energy for which $h_{c}=h_{c}^{+}$. The minimum of the energies attained as $h_{c}$ traverses the indicated interval provides the asserted local minimum that does not minimize globally. In view of the left hand inequality in (4.1), the surface $\mathcal{S}$ at this local minimizing configuration meets $\mathcal{B}$ in the angle $\gamma$ determined by $\mathrm{e}_{2}-\mathrm{e}_{1}=\mathrm{e}_{0} \cos \gamma$, see Theorem 1.2.

Finally we observe that the case $\rho<\rho_{1}<\rho_{2}$ is subsumed under the case just discussed, by reversing the direction of gravity. The substance of the reasoning is identical.

Remark 1. The method for Theorem 4.1 imposes the requirement $e_{2}>e_{1}$, which implies by (3.1) that $\gamma>\pi / 2$. A work now in preparation provides explicit examples of floating equilibria with $\rho_{1}<\rho_{2}<\rho$ and for which $\gamma<\pi / 2$, and thus we see that the criterion of the theorem suffices for floating but is not necessary.

Remark 2. The case of a general convex body in three dimensions leads to some significant distinctions in the announced results. That work will appear in an independent article joint with Vogel [17].

\section{Acknowledgements}

I am indebted to the Max-Planck-Institut für Mathematik in den Naturwissenschaften, in Leipzig, for its hospitality during preparation of this work. I wish especially to thank John McCuan for very helpful comments that have led to a number of improvements in the exposition.

Open Access. This article is distributed under the terms of the Creative Commons Attribution Noncommercial License which permits any noncommercial use, distribution, and reproduction in any medium, provided the original author(s) and source are credited.

\section{References}

[1] Laplace, P.S., Traité de mécanique céleste: supplement 2, 909-945, au Livre X. In: Euvres Complète, vol. 4. GauthierVillars, Paris. See also the annotated English translation by N. Bowditch (1839), reprinted by Chelsea, New York, 1966 $(1805 / 1806)$ 
[2] Raphaël, E., di Meglio, J.-M., Berger, M., Calabi, E.: Convex particles at interfaces. J. Phys. I France 2, 571-579 (1992)

[3] Gifford, W.A., Scriven, L.E.: On the attraction of floating particles. Chem. Eng. Sci. 26, 287 (1971)

[4] Kralchevsky, P.A., Nagayama, J.: Capillary interactions between particles bound to interfaces, liquid films and biomembranes. Adv. Coll. Interface Sci. 85, 145-192 (2000)

[5] Mansfield, E.H., Sepangi, H.R., Eastwood, E.A.: Equilibrium and mutal attraction or repulsion of objects supported by surface tension. Phil. Trans. R. Soc. Lond. A 355, 869-919 (1997)

[6] Bowden, N., Oliver, S.R.J., Whitesides, G.M.: Mesoscale self-assembly: capillary bonds and negative menisci. J. Phys. Chem. B 104, 2714-2724 (2000)

[7] Finn, R.: Floating and partly immersed balls in a weightless environment. Funct. Differ. Equ. 12(1-2), 167-173 (2005)

[8] Finn, R.: The contact angle in capillarity. Phys. Fluids 18(4), 047102 (2006).

[9] Bhatnagar, R., Finn, R.: Equilibrium configurations of an infinite cylinder in an unbounded fluid. Phys. Fluids 18(4), 047103 (2006).

[10] Finn, R.: Floating bodies subject to capillary attractions. J. Math. Fluid Mech. (online first)

[11] Finn, R., Sloss, M.: Floating bodies in neutral equilibrium. J. Math. Fluid Mech. (online first)

[12] McCuan, J.: A variational formula for floating bodies. Pac. J. Math. 231(1), 167-191 (2007)

[13] Young, T.: An essay on the cohesion of fluids. Philos. Trans. Roy. Soc. London 95, 65-87 (1805), Reprinted with some author's alterations and additions in Peacock, G. (ed.), Miscellaneous Works, Vol. 1, John Murray, London, 1855

[14] Bakker, G.: Kapillarität und Oberflächenspannung. In: Handbuch der Experimentalphysik. Akademische Verlagsgesellschaft, Leipzig (1928)

[15] Vogel, T.I.: Symmetric unbounded liquid bridges. Pac. J. Math 103(1), 205-241 (1982)

[16] Finn, R.: Capillary surfaces. In: Encyclopedia of Mathematical Physics, pp. 431-445. Elsevier, Amsterdam (2006)

[17] Finn, R., Vogel, T.I.: Floating bodies in three dimensions. Analysis 29(2), 125-140 (2009)

Robert Finn

Mathematics Department

Stanford University

Stanford, CA 94305-2125, USA

e-mail: finn@math.stanford.edu

(accepted: June 12, 2009; published online: October 2, 2009) 